\title{
RECURSIVE CONSTRUCTIONS FOR EQUIDISTANT PERMUTATION ARRAYS
}

\author{
P. J. SCHELLENBERG and S. A. VANSTONE \\ (Received 21 March 1977) \\ Communicated by W. D. Wallis
}

\begin{abstract}
An equidistant permutation array (EPA) is a $v \times r$ array defined on an $r$-set, $R$, such that

(i) each row is a permutation of the elements of $R$ and

(ii) any two distinct rows agree in $\lambda$ positions (that is, the Hamming distance is $(r-\lambda)$ ).

Such an array is said to have order $v$. In this paper we give several recursive constructions for EPA's.

The first construction uses a resolvable regular pairwise balanced design of order $v$ to construct an EPA of order $v$. The second construction is a generalization of the direct product construction for Room squares.

We also give a construction for intersection permutation arrays, which arrays are a generalization of EPA's.
\end{abstract}

Subject classification (Amer. Math. Soc. (MOS), 1970): 05 B 30

\section{Introduction}

An equidistant permutation array (EPA), $\mathrm{A}(r, \lambda ; v)$, is a $v \times r$ array defined on the elements of an $r$-set, $R$, such that

(i) each row is a permutation of the elements of $R$ and

(ii) any two rows agree in $\lambda$ positions (that is, the Hamming distance between any two rows is $(r-\lambda)$ ).

Let $R(r, \lambda)$ be the maximum value for $v$ such that there exists an $\mathrm{A}(r, \lambda ; v)$. Deza (1976) has shown that $R(r, \lambda) \leqslant \max \left(\lambda+2, n^{2}+n+1\right)$, where $n=r-\lambda$. Mullin and Nemeth (to appear) have shown $R(r, 1) \leqslant r(r-4)-1$ for $r>6$. Recently, Heinrich and van Rees (to appear) have shown that $R(r, 1) \geqslant 2 r-4$ for $r>5$. This implies

$$
R(r, \lambda) \geqslant 2(r-\lambda)-2 \text { for } r>5 \text {. }
$$

An EPA is said to be $k$-uniform, for some fixed natural number $k$, or, more simply, uniform if each column of the array contains every element of $R$ either $k$ times or zero times. For example, a Latin square of order $n$ is an $\mathrm{A}(n, 0 ; n)$. It is also uniform since each element is contained in each column precisely once. In this paper, recursive constructions for EPA's and for uniform EPA's are described. These constructions are established using the language of regular 
pairwise balanced designs and of generalized Room squares, both of which we now lefine.

An $(r, \lambda)$-design is a collection, $B$, of subsets, called blocks, of a set $V$, whose slements are called varieties, such that

(i) each variety is contained in $r$ blocks and

(ii) each (unordered) pair of varieties is contained in $\lambda$ blocks.

$(r, \lambda)$-designs are also called regular pairwise balanced designs. An $(r, \lambda)$-design is said to be resolvable if the blocks of $B$ can be partitioned into $r$ sets, $B_{1}, B_{2}, \ldots, B_{r}$, called resolution classes, such that each variety is contained in precisely one block of each resolution class. A resolvable $(r, \lambda)$-design is said to be orthogonally resolvable if the blocks can be partitioned into one collection of resolution classes, $R_{1}, R_{2}, \ldots, R_{r}$, and into a second collection of resolution classes $C_{1}, C_{2}, \ldots, C_{r}$, such that, for any $i, j=1,2, \ldots, r,\left|R_{i} \cap C_{j}\right|=0$ or 1 . An orthogonally resolvable $(r, \lambda)$-design defined on the elements of a $v$-set is denoted by $\mathrm{OD}(r, \lambda ; v)$ or, more simply, by OD. Deza, Mullin and Vanstone (1976) have shown that the existence of an $\mathrm{OD}(r, \lambda ; v)$ is equivalent to the existence of an $\mathrm{A}(r, \lambda ; v)$. An $\mathrm{OD}(r, \lambda ; v)$ is said to be $k$-uniform or, more simply, uniform if every block has cardinality $k$.

A generalized Room square (GRS), $\mathrm{S}(r, \lambda ; v)$, of side $r$, index $\lambda$ and order $v$ is an $r \times r$ array of elements from a $v$-set, $V$, such that

(i) each cell of the array contains a subset (possibly empty) of $V$,

(ii) the subsets in each row and in each column are a partition of $V$ and

(iii) every pair of elements of $V$ is contained in precisely $\lambda$ of the cells of the array.

Deza, Mullin and Vanstone (1976) have shown that the existence of an $S(r, \lambda ; v)$ is equivalent to the existence of an $\mathrm{A}(r, \lambda ; v)$ and an $\mathrm{OD}(r, \lambda ; v)$.

A Room square is an $\mathrm{S}(2 n-1,1 ; 2 n)$ in which every non-empty subset has cardinality 2 . Room squares have been extensively studied and it is known (see, for example, Mullin and Wallis, 1975) that there is a Room square of side $2 n-1$ for all positive integers $n$ except $n=2,3$. There is no Room square of side 3 nor of side 5. This implies there exists an $\mathrm{A}(2 n-1,1 ; 2 n)$ for all positive integers $n>3$, as observed by Deza, Mullin and Vanstone (1976).

An $\mathrm{S}(r, \lambda ; v)$ is said to be $k$-uniform or, more simply, uniform if every non-empty subset in the array has cardinality $k$.

Since the non-empty cells of a Room square are pairs, there exists a two-uniform $\mathrm{S}(2 n-1,1 ; 2 n)$ for $2 n-1>5$ and thus a two-uniform $\mathrm{A}(2 \mathrm{n}-1,1 ; 2 n)$ for $2 n-1>5$.

An $\mathrm{S}(r, \lambda ; v)$ is said to be full if it has no empty cells. A Latin square of order $n$ is a full $\mathrm{S}(n, 0 ; n)$. An $\mathrm{OD}(r, \lambda ; v)$ is said to be full if each resolution class contains $r$ blocks or, equivalently, if $|B|=r^{2}$. An $\mathrm{A}(r, \lambda ; v)$ is said to be full if every column contains all $v$ of the elements. It is conjectured that if an $\mathrm{S}(r, \lambda ; v)$ is full, then it is an $\mathrm{S}(r, 0 ; r)$; that is, it is a Latin square of order $r$. 


\section{A recursive construction for uniform EPA's}

Suppose we have a resolvable $(r, \lambda)$-design, say $R$, on the set $V$ such that no resolution class has more than $t$ blocks. Let $S$ be an $(s, \mu)$-design on a $t$-set $T=\{1,2, \ldots, t\}$. From these two designs we can construct an $(r s, \lambda s+(r-\lambda) \mu)$ design, say $U$, on the set $V$ as follows. Let $A_{1}, A_{2}, \ldots, A_{8}, s \leqslant t$, be the blocks of any resolution class of $R$. Let $A_{s+1}=A_{s+2}=\ldots=A_{i}=\varnothing$. We construct a new block corresponding to each block, $B$, of $S$; namely, the block

$$
\bigcup_{i \in B} A_{i} \text {. }
$$

This is repeated for each resolution class of $R$. The resultant set of blocks can be shown to constitute $U$, an $(r s, \lambda s+(r-\lambda) \mu)$-design on the set $V$.

It can also be shown that if $S$ is resolvable, so is $U$. Furthermore, if $S$ is an OD, then so is $U$. Thus we have

THEOREM 2.1. If there is a resolvable $(r, \lambda)$-design, $R$, on variety set $V$ with at most $t$ blocks in any resolution class and if there is an orthogonally resolvable $(s, \mu)$ design, $S$, on $t$ varieties then there is an orthogonally resolvable $(r s, \lambda s+(r-\lambda) \mu)$ design on variety set $V$.

COROLLARY 2.2. If the blocks of $R$ all have the same cardinality (that is, if $R$ is a $B I B D)$, and if $S$ is a uniform $O D$, then $U$ is a uniform $O D$ as well.

This follows immediately from Theorem 2.1. The above construction and its applications have been studied by Lonz and Vanstone (unpublished manuscript).

TheOREM 2.3. If $n=2^{k}>4$, then there is a $2 n$-uniform $\operatorname{OD}\left(n^{2}-1,2 n-1 ; n^{2}\right)$.

Proof. For $n=2^{k}>4$, there is a BIBD with parameters $\left(n^{2}, n^{2}+n, n+1, n, 1\right)$, namely, the affine plane of order $n$. Since $n$ is even and greater than 6 , there is a uniform $\mathrm{OD}(n-1,1 ; n)$; namely a Room square of order $n$. Thus, by Corollary 2.2, there is a $2 n$-uniform $\mathrm{OD}\left(n^{2}-1,2 n-1 ; n^{2}\right)$.

We define a $v \times r$ intersection permutation array (IPA), $\mathrm{A}_{t}(r, \lambda ; v)$, of degree $t$ and index $\lambda$ to be a set of $v$ permutations on the symbols $1,2, \ldots, r$ such that any $t$ of the permutations agree in precisely $\lambda$ positions. Clearly an IPA of degree 2 is an EPA. Not every IPA is an EPA as is demonstrated in the following example.

$\begin{array}{ll}15342687 & \\ 12543876 & \\ 12354768 & A_{3}(8,1 ; 5) \\ 52341678 & \\ 14235678 & \end{array}$

This is a $5 \times 8$ IPA of degree 3 and index 1 , but it is not an EPA. 
Let $v=\mathrm{R}_{t}(r, \lambda)$ be the maximum value of $v$ such that there exists an $\mathrm{A}_{d}(r, \lambda ; v)$. To the best of our knowledge, this function has not been investigated.

A regular t-wise balanced design, $\mathrm{D}_{l}\left(r, \lambda_{l} ; v\right)$ is a collection of subsets, called blocks, of a $v$-set, $V$, in which every variety is contained in $r$ blocks and every $t$-set of varieties is contained in $\lambda_{t}$ blocks. Let $\mathrm{OD}_{t}\left(r, \lambda_{t} ; v\right)$, or more simple $\mathrm{OD}_{t}$, denote an orthogonally resolvable regular $\boldsymbol{t}$-wise balanced design. A $\boldsymbol{k}$-uniform, or more simply a uniform, $\mathrm{D}_{t}\left(r, \lambda_{t} ; v\right)$ is a $t$-design (see Hughes, 1965) with parameters $t-\left(v, k, \lambda_{l}\right)$. Since every block of a $k$-uniform $\mathrm{D}_{t}\left(r, \lambda_{l} ; v\right), R$, has cardinality $k$, it follows (Hughes, 1965) that, for $1<s<t, R$ is also a $k$-uniform $\mathrm{D}_{s}\left(r, \lambda_{s} ; v\right)$ where

$$
\lambda_{s}=\lambda_{l}\left(\begin{array}{c}
v-s \\
t-s
\end{array}\right) /\left(\begin{array}{c}
k-s \\
t-s
\end{array}\right) \text {. }
$$

The corresponding result for non-uniform $\mathrm{D}_{l}\left(r, \lambda_{l} ; v\right)$ 's is not in general true.

The following theorem describes a construction for $\mathrm{OD}_{3}$ 's.

THEOREM 2.4. If there is a resolvable $\mathrm{D}_{3}\left(r, \lambda_{3} ; v\right), R$, which is also a $\mathrm{D}_{2}\left(r, \lambda_{2} ; v\right)$ and each resolution class has cardinality at most $t$ and if there is an $\mathrm{OD}_{3}\left(s, \mu_{3} ; t\right), S$, which is also an $\mathrm{OD}_{2}\left(s, \mu_{2} ; t\right)$ then there is an $\mathrm{OD}_{3}\left(r s, \eta_{3} ; v\right), U$, which is also an $\mathrm{OD}_{2}\left(r s, \eta_{2} ; v\right)$ where

and

$$
\eta_{3}=\lambda_{3} s+3\left(\lambda_{2}-\lambda_{3}\right) \mu_{2}+\left(r-3 \lambda_{2}+2 \lambda_{3}\right) \mu_{3}
$$

$$
\eta_{2}=\lambda_{2} s+\left(r-\lambda_{2}\right) \mu_{2}
$$

Proof. We use the construction described above which was used to establish Theorem 2.1. Theorem 2.1 implies $U$ is an $\mathrm{OD}_{2}\left(r s, \eta_{2} ; v\right)$.

Now consider any three varieties of $R$, say $\{a, b, c\}$. There are $\lambda_{3}$ blocks which contain all three of these elements. The corresponding $\lambda_{3}$ resolution classes, in turn, give rise to $\lambda_{3} s$ blocks in $U$ which contain all three of $a, b$ and $c$.

Now consider those resolution classes of $R$ in which no block contains all three of $a, b$ and $c$ but some block contains a pair of them. The number of such resolution classes is $3\left(\lambda_{2}-\lambda_{3}\right)$. These resolution classes give rise to $3\left(\lambda_{2}-\lambda_{3}\right) \mu_{2}$ blocks in $U$ which contain all three of $a, b$ and $c$.

Finally, we have those resolution classes in which $a, b$ and $c$ are in distinct blocks. The number of such resolution classes is $\left(r-3 \lambda_{2}+2 \lambda_{3}\right)$ and these classes, in turn, give rise to $\left(r-3 \lambda_{2}+2 \lambda_{3}\right) \mu_{3}$ blocks of $U$ which contain all three of $a, b$ and $c$.

Thus, $U$ is also an $\mathrm{OD}_{3}\left(r s, \eta_{3} ; v\right)$ with

$$
\eta_{3}=\lambda_{3} s+3\left(\lambda_{2}-\lambda_{3}\right) \mu_{2}+\left(r-3 \lambda_{2}+2 \lambda_{3}\right) \mu_{3} \text {. }
$$

This completes the proof.

COROLlARY 2.5. If there is a resolvable $k$-uniform $\mathrm{D}_{3}\left(r, \lambda_{3} ; v\right), R$, and if there is an $l$-uniform $\mathrm{OD}_{3}\left(s, \mu_{3} ; t\right), S$, where $t=v / k$, then there is a $k l$-uniform $\mathrm{OD}_{3}\left(r s, \eta_{3} ; v\right), U$. 
Proof. Hughes (1965) has shown $R$ is a $k$-uniform $\mathrm{D}_{2}\left(r, \lambda_{2} ; v\right)$ where $\lambda_{2}=\lambda_{3}(v-2) /(k-2)$ and $S$ is an $l$-uniform $\operatorname{OD}_{2}\left(s, \mu_{2} ; t\right)$ where $\mu_{2}=\mu_{3}(t-2) /(l-2)$. Thus, by Theorem $2.4, U$ is a $k l$-uniform $\mathrm{OD}_{3}\left(r s, \eta_{3} ; v\right)$ with $\eta_{3}$ as defined above. This completes the proof.

We now use this corollary to establish

THEOREM 2.6. If $v=2 t k>6 k$, then there is a $2 k$-uniform

$$
\mathrm{OD}_{3}\left(\left(\begin{array}{l}
v-1 \\
k-1
\end{array}\right)(2 t-1), \eta_{3} ; v\right)
$$

where

$$
\eta_{3}=\left(\begin{array}{l}
v-3 \\
k-3
\end{array}\right)(2 t-4)+3\left(\begin{array}{l}
v-2 \\
k-2
\end{array}\right)
$$

Proof. If $v=2 t k$ there is a resolvable $k$-uniform

$$
\mathrm{D}_{3}\left(\left(\begin{array}{l}
v-1 \\
k-1
\end{array}\right),\left(\begin{array}{l}
v-3 \\
k-3
\end{array}\right) ; v\right)
$$

consisting of all $k$-sets of a $v$-set (Baranyai, 1975). Each resolution class has $2 t$ blocks. Since $2 t>6$, there is a Room square of side $2 t-1$ which is a two-uniform $\mathrm{OD}_{3}(2 t-1,0 ; 2 t)$. Corollary 2.5 now yields the result.

\section{Multiplying orders}

In this section we describe a recursive construction for generalized Room squares in which the order of the resultant square is the product of the orders of the two component squares. This construction is a generalization of the recursive construction of Stanton and Horton (1972) for Room squares.

We begin by establishing some notation. Suppose $L_{1}, L_{2}, \ldots, L_{m}$ is a set of pairwise orthogonal Latin squares (POLS) of order $s$ (see Ryser, 1963). Let $L_{1} \oplus L_{2} \oplus \ldots \oplus L_{m}$ denote the array obtained if the $m$ POLS are superimposed to form a single $s \times s$ array in which each cell contains an ordered set of $m$ elements. If $L$ is a Latin square of order $s$ and $p$ is an arbitrary symbol, let $(L, p)$ denote the Latin square of order $s$ obtained from $L$ by replacing each element $a$ in $L$ by the ordered pair $(a, p)$. If $R$ is a generalized Room square and $p$ is an arbitrary symbol, let $(R, p)$ denote the generalized Room square obtained from $R$ by replacing each element $a$ of $R$ by the ordered pair $(a, p)$. Finally a generalized Room square $\mathrm{S}(r, \lambda ; v)$ is said to have a complete set of singletons if there exist $v$ cells in the array each containing a single element with no two cells containing the same element. 
THEOREM 3.1. If there is an $\mathrm{S}(r, \lambda ; v), T$, which contains a complete set of singletons and in which no cell has more than $m$ elements, if there is an $\mathrm{S}(s, \lambda ; s), U$, and if there are $m$ POLS of order $s, L_{1}, L_{2}, \ldots, L_{m}$, then there is an $\mathrm{S}(r s, \lambda ; v s), V$.

Proof. Let $U, L_{1}, L_{2}, \ldots, L_{m}$ all be defined on the same set of elements. $V$ is constructed from $T$ by replacing each cell of $T$ by an $s \times s$ array as follows. Each empty cell of $T$ is replaced by an $s \times s$ array of empty cells. The cell of the complete set of singletons containing $a$ is replaced by the $s \times s$ array $(U, a)$. Every other cell contains a subset of elements. If $\left\{a_{1}, a_{2}, \ldots, a_{r}\right\}$ is in cell $(i, j)$, replace this cell by $\left(L_{1}, a_{1}\right) \oplus\left(L_{2}, a_{2}\right) \oplus \ldots \oplus\left(L_{r}, a_{r}\right)$.

Clearly each row and each column of $V$ contains every element of $V$. Suppose $a$ and $b$ are any two distinct elements from $T$ and suppose $x$ and $y$ are two (not necessarily distinct) elements from $U .\{a, b\}$ is contained in $\lambda$ cells of $T$. Suppose that in one such cell $a$ and $b$ are replaced by $\left(L_{i}, a\right)$ and $\left(L_{j}, b\right), i \neq j$. Since $L_{i}$ and $L_{j}$ are orthogonal $L_{i} \oplus L_{j}$ contains the pair $(x, y)$. Thus $\left(L_{i}, a\right) \oplus\left(L_{j}, b\right)$ contains $((x, a),(y, b))$. Thus each pair $\{(x, a),(y, b)\}, a \neq b$, is in the array $\lambda$ times. Now suppose $x$ and $y$ are two distinct elements of $U .\{x, y\}$ is contained in $\lambda$ cells of $U$. Thus, for any $a$ of $T,\{(x, a),(y, a)\}$ is contained in $\lambda$ cells of $(U, a)$. Thus $V$ is an $\mathrm{S}(r s, \lambda ; v s)$.

Heinrich and van Rees (to appear) have shown that if $n>5$, there is an $\mathrm{S}(n, 1 ; 2 n-4)$ with a complete set of singletons and the largest block is of length $n-2$. For any odd integer $s$ such that there are $n-2$ POLS of side $s$, there is an $\mathrm{S}(s, 1 ; s)$, namely one obtained by deleting a variety from the Room square of side $s$. Thus by Theorem 3.1, there is an $\mathrm{S}(n s, 1 ; 2 n s-4 s)$. Now, the Heinrich-Rees result implies the existence of an $S(n s, 1 ; 2 n s-4)$ whose order is greater than $2 n s-4 s$ which suggests that Theorem 3.1 is of little or no use. However, it could conceivably be of great use. For example, at present it is not known if there is an $\mathrm{S}(n, 1 ; 2 n)$ for any integer $n>1$. McCarthy and Mullin (unpublished manuscript) have shown that $\mathrm{R}(7,1)=13$ and $\mathrm{R}(8,1) \geqslant 15$. If just one $\mathrm{S}(n, 1 ; 2 n)$ with a complete set of singletons is constructed (say by a computer search) then Theorem 3.1 would imply the existence of an $\mathrm{S}(n, 1 ; 2 n)$ for an infinity of values of $n$.

\section{Some direct constructions}

We conclude this paper by listing briefly some direct constructions for EPA's which appear in the literature. These results establish lower bounds for $\mathrm{R}(r, \lambda)$.

Heinrich and van Rees (to appear) use self-orthogonal Latin squares to establish

THEOREM 4.1. For $r>5$, there exists an $\mathrm{A}(r, 1 ; 2 r-4)$.

Heinrich, van Rees and Wallis (preprint) use sets of POLS to establish

THEOREM 4.2. If there are $\lambda+2$ POLS of order $n$, then there is an

$$
A(n+\lambda+2, \lambda ;(\lambda+1) n+1) \text {. }
$$


The final result quoted here is a generalization by Vanstone (to appear) of a result of Woodall (unpublished manuscript). A group divisible design,

$$
\mathrm{GD}\left(v ; k, m ; \lambda_{1}, \lambda_{2}\right) \text {, }
$$

is a collection of $k$-subsets, called blocks, of a $v$-set, $V$, whose elements are called varieties, such that

(a) $V$ is partitioned into $m$-subsets, called groups, and

(b) any pair of varieties from the same group is contained in $\lambda_{1}$ blocks and any pair of varieties from different groups is contained in $\lambda_{2}$ blocks.

For the following result we require orthogonally resolvable (see Section 1) group divisible designs. However, the blocks of these designs need not all have the same cardinality nor need the groups all be of the same cardinality. Let us denote such an orthogonally resolvable group divisible design by $\operatorname{OGD}\left(r ; \lambda_{1}, \lambda_{2} ; P, v\right)$ where $r$ is the number of resolution classes and

$$
P=\left\{P_{1}, P_{2}, \ldots, P_{s}\right\}
$$

represents the partition of $V$ into $P_{1}, P_{2}, \ldots, P_{g}$.

THEOREM 4.3. If there is an OGD $\left(r_{1} ; \lambda_{1}, \lambda_{2} ; P, v\right)$ and an $\operatorname{OGD}\left(r_{2} ; \mu_{1}, \mu_{2} ; P, v\right)$ such that $\lambda_{1}+\mu_{1}=\lambda_{2}+\mu_{2}$, then there is an $\mathrm{A}\left(r_{1}+r_{2}, \lambda_{1}+\mu_{1} ; v\right)$.

Proof. The union of these two designs is an $\operatorname{OD}\left(r_{1}+r_{2}, \lambda_{1}+\mu_{1} ; v\right)$.

Woodall (unpublished manuscript) uses a complete set of POLS of order $n$ (that is, a set of $(n-1)$ such POLS) to construct an $\operatorname{OGD}(3 n ; 0,3 ; P, n(n-1))$. In this design each group has cardinality $n$. He uses the design consisting of all $(n-2)$ subsets of an $(n-1)$ set to construct an $\operatorname{OGD}(n ; n, n-3 ; P, n(n-1))$. Then Theorem 4.3 implies

THEOREM 4.4. If $n$ is a prime power, there is an $\mathrm{A}(4 n, n ; n(n-1))$.

Vanstone (to appear) uses finite projective geometries to construct two orthogonally resolvable group divisible designs with the required properties to establish

THEOREM 4.5. If $q$ is a prime power and $n$ is a positive integer, there exists an

$$
\mathrm{A}\left(\frac{3 q\left(q^{n-1}-1\right)}{q-1}+q, \frac{3 q\left(q^{n-2}-1\right)}{q-1}+q ; q^{n-1}(q-1)\right) .
$$

Observe that for $n=2$, Theorem 4.5 is precisely Theorem 4.4 .

\section{References}

Z. Baranyai (1975), On the factorization of the Complete Uniform Hypergraph, Infinite and Finite Sets, Vol. 1 (North Holland Publishing Co., Budapest, Hungary), pp. 91-108.

M. Deza (1976), "Matrices dont deux lignes quelconques coincident dans un nombre donné de positions communes", J. Combinatorial Theory $(A)$ 20, 306-318. 
M. Deza, R. C. Mullin and S. A. Vanstone (1976), "Room squares and equidistant permutation arrays", Ars Combinatoria, 2, 235-244.

Katherine Heinrich and G. H. J. van Rees (to appear), "Some constructions for equidistant permutation arrays of index one", Utilitas Mathematica.

Katherine Heinrich, G. H. J. van Rees and W. D. Wallis (preprint), "A general construction for equidistant permutation arrays".

D. R. Hughes (1965), "On $t$-designs and groups", Amer. J. Math. 87, 761-778.

R. C. Mullin and E. Nemeth (to appear), "An improved bound for equidistant permutation arrays of index one", Utilitas Math.

R. C. Mullin and W. D. Wallis (1975), "The existence of Room squares", Aequationes Math. 13, 1-7.

H. J. Ryser (1963), Combinatorial Mathematics, The Carus Mathematical Monographs 14 (Mathematical Association of America).

R. G. Stanton and J. D. Horton (1972), “A multiplication theorem for Room squares", J. Combinatorial Theory 12, 322-325.

S. A. Vanstone (to appear), "Pairwise orthogonal generalized Room squares and equidistant permutation arrays", J. Combinatorial Theory $(A)$.

University of Waterloo

Waterloo, Ontario 